\title{
. \\ A wireless radiofrequency-powered insect-scale flapping-wing aerial vehicle
}

\author{
Takashi Ozaki@®, Norikazu Ohta, Tomohiko Jimbo $₫$ and Kanae Hamaguchi
}

\begin{abstract}
Insect-scale aerial vehicles are useful tools for communication, environmental sensing and surveying confined spaces. However, the lack of lightweight high-power-density batteries has limited the untethered flight durations of these micro aerial vehicles. Wireless power transmission using radiofrequency electromagnetic waves could potentially offer transmissivity through obstacles, wave-targeting/focusing capabilities and non-mechanical steering of the vehicles via phased-array antennas. But the use of radiofrequency power transmission has so far been limited to larger vehicles. Here we show that a wireless radiofrequency power supply can be used to drive an insect-scale flapping-wing aerial vehicle. We use a sub-gram radiofrequency power receiver with a power-to-weight density of $4,900 \mathrm{~W} \mathrm{~kg}$, which is five times higher than that of off-the-shelf lithium polymer batteries of similar mass. With this system, we demonstrate the untethered take off of the flapping-wing micro aerial vehicle. Our RF-powered aircraft has a mass of $1.8 \mathrm{~g}$ and is more than $\mathbf{2 5}$ times lighter than previous radiofrequency-powered micro aerial vehicles.
\end{abstract}

M icro aerial vehicles (MAVs) are of potential use in environmental sensing ${ }^{1}$, communication ${ }^{2,3}$, indoor entertainment $^{4}$, search and rescue ${ }^{5}$, and the autonomous inspection of confined or dangerous areas ${ }^{6}$. However, MAV regulations are being tightened due to the risk of accidents from crashing MAVs. In the USA, for example, drones weighing over $250 \mathrm{~g}$ must be registered, and drone flight areas are heavily restricted ${ }^{7}$. Japan has also recently announced that the threshold weight of MAVs subject to flight restrictions will be reduced from 200 to $100 \mathrm{~g}$ (ref. ${ }^{8}$ ). These intensified requirements demand smaller and lighter MAVs. In particular, as the collision energy of a falling aerial vehicle strongly depends on the vehicle mass, the safety of MAVs can be increased by designing and developing lightweight insect-scale MAVs.

The critical components in developing a fully untethered insect-scale MAV are the actuator, drive electronics and power supply. Although larger-scale MAVs generally employ electromagnetic motors as actuators, their efficiency is notably reduced when the vehicle size is decreased due to friction and resistance losses 9 . There are several other actuation mechanisms, including electrostatic, shape memory alloy and piezoelectric mechanisms. Considering the high speed and high output required for flights, piezoelectric actuation is the most promising mechanism ${ }^{10}$ and is commonly employed. As piezoelectric actuation generates reciprocal motion, a bioinspired flapping-wing mechanism is more suitable for insect-scale MAVs than a rotating propeller ${ }^{11-15}$. Lightweight, high-voltage and high-power electronics for piezoelectric actuation have also been studied ${ }^{16-18}$. However, the low energy density of conventional batteries makes them unsuitable for insect-scale MAVs ${ }^{19}$.

The most successful work of a battery-powered MAV is the DelFly ${ }^{20,21}$. DelFly Nimble is, for example, a bird-scale $29 \mathrm{~g}$ tailless vehicle capable of hovering and moving in all directions ${ }^{21}$. DelFly Micro is an insect-scale model with a mass of $3 \mathrm{~g}$ (ref. ${ }^{20}$ ). However, this vehicle can only fly forward with the help of lift during translational movement and has no hovering capability. A similar vehicle ( $3.2 \mathrm{~g}$, battery powered) has been developed with a maximum thrust force (4 gram-force (gf)) that exceeds its mass, but it lacks the additional actuators necessary for controlled flight ${ }^{22}$. At the insect scale, there are currently no battery-powered vehicles capable of both controlled flight and hovering by supplying enough lift to support their own weight. Thus, alternative energy sources with higher power density are required to realize insect-scale MAVs that can fly freely.

The flight time of a bird-scale battery-powered MAV is usually less than $10 \mathrm{~min}$. Therefore, even if an insect-scale battery-powered MAV can be created, it would be expected to have a short flight duration. To overcome this drawback, wireless power transmission via lasers or light has been developed ${ }^{23,24}$. However, for indoor applications or in confined spaces, laser/light power transmission for small MAVs has serious limitations. Laser/light power transmission is easily blocked by obstacles, and fast and robust laser steering technology (for example, phased-array lasers ${ }^{25,26}$ ) is still not readily available off the shelf. In contrast, radiofrequency (RF) electromagnetic waves can penetrate insulating obstacles or objects smaller than their wavelength, as demonstrated by mobile phone communications. These characteristics provide considerable advantages for indoor applications. In addition, beam-steering technology implemented by a phased-array system is very practical ${ }^{27-29}$. Previous research on MAVs with RF power transmission has been limited to large multirotor vehicles weighing over a few dozen grams ${ }^{30-34}$, and the feasibility of RF power transmission for insect-scale MAVs is still uncharted.

Beyond MAVs, there are earlier reports on RF power transmission to insect-scale robots ${ }^{35,36}$. In particular, a walking robot powered by a piezoelectric actuator with a mass of $2.1 \mathrm{~g}$ has been developed $^{35}$. The power-receiving circuit in this robot has a maximum power output of $1 \mathrm{~W}$ and a mass of $200 \mathrm{mg}$, which may be applicable for the flight of an MAV of equivalent weight. However, the power transmission technology used in these studies is based on near-field electromagnetic waves; the robot cannot be far away from the transmitter. To transmit power to MAVs that can fly freely, a system using radiative, far-field electromagnetic waves is desirable.

In this Article, we report a sub-gram RF power receiver for $5 \mathrm{GHz}$ far-field radiative electromagnetic waves with a power density that is sufficient for flight. We install the electronics on an insect-scale flapping-wing vehicle (Fig. 1a); the piezoelectric 
a

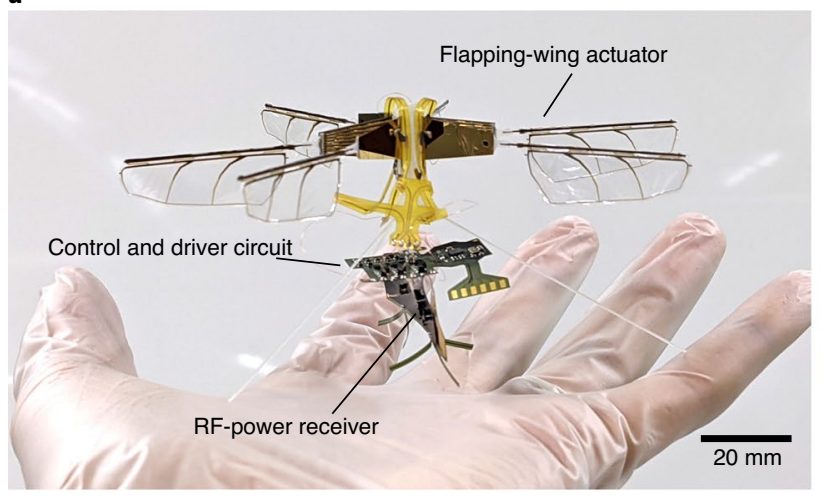

b

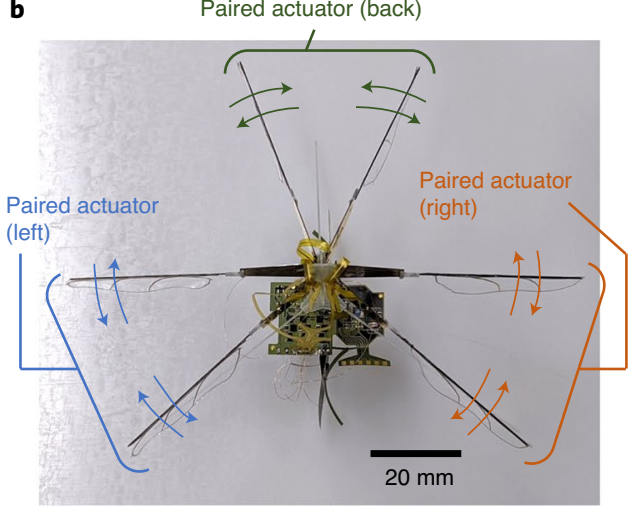

C

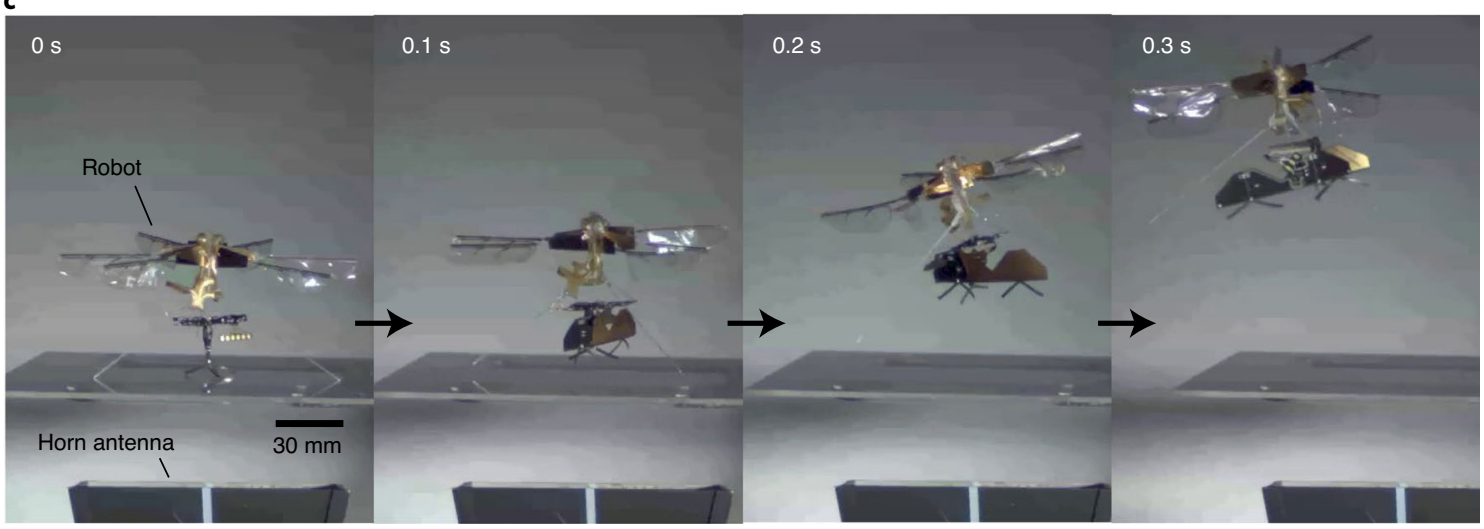

Fig. 1 | Integrated MAV with RF power receiver. a, Photograph of the fabricated aircraft. b, Configuration of flapping-wing actuators. c, Flight demonstration.

flapping-wing actuators are on the upper half of the vehicle and the electronics are mounted on the lower half. The electronics consist of three sets of $5 \mathrm{GHz}$ dipole power-receiving antennas/rectifiers, a high-voltage three-channel driver for the flapping-wing actuators and a microprocessor. The vehicle has six independently actuated wings that are actuated in pairs (Fig. 1b). For a configuration with the same direction of motion in actuators and wings, this paired actuation scheme results in a low vibration energy loss and high lift force efficiency ${ }^{37,38}$. Level flight can be achieved by adjusting the lift forces of the three pairs of actuator, and the vehicle can be successfully launched by the RF power supply with a transmission power of $30 \mathrm{~W}$ (Fig. 1c and Supplementary Video 1). Our vehicle is more than 25 times lighter than the previous vehicles powered by RF (Extended Data Table 1).

\section{Power-receiving electronic system}

Photographs and system schematic of the developed high-powerdensity, lightweight electronics are shown in Fig. 2a,b, respectively. The circuit was fabricated using standard mass-production flexible printed circuit (FPC) technology. Thermal management is a key factor for achieving a high power density because any concentrated overheating results in high power loss as well as destruction of the microscale electrical components. To distribute the power-loss heat, a multi-antenna/rectifier configuration was employed; the $5 \mathrm{GHz}$ electromagnetic wave is received by three dipole antennas and converted to direct current (d.c.) power by three rectifiers (the schematic is shown in Extended Data Fig. 1a). The dipole antennas are fabricated from the FPC wiring of $\mathrm{Cu}$ patterns, such that the mass of the antenna is only $4 \mathrm{mg}$. The maximum power-receiving efficiency of the rectifier was approximately 67\% (Extended Data Fig. 1c).
Because the voltage of the received electrical power can exceed the maximum input voltage of the digital components, that is, the microprocessor, it should be regulated to a digital-level voltage. We separated part of the electrical power and adjusted it to $3.3 \mathrm{~V}$ using a voltage regulator for the microprocessor (CC2640R2F, Texas Instruments). The remaining electrical power was also regulated with an upper limit of $6.0 \mathrm{~V}$, which is the maximum input voltage of the $3.3 \mathrm{~V}$ voltage regulator. Next, the power source was boosted to yield a constant high voltage ( $175 \mathrm{~V}$ maximum) using a d.c.-d.c. converter (the schematic is shown in Extended Data Fig. 1b), as required for the piezoelectric drive. Then, it was reshaped into three square-wave actuation signals by a three-channel high-voltage driver controlled by the microprocessor (schematic of the microprocessor and high-voltage driver are shown in Extended Data Fig. 2a,b, respectively; the driving waveform is illustrated in Extended Data Fig. 3; connection diagram of the subcircuits is shown in Extended Data Fig. 2c).

Note that the high output voltage is restricted by the limit of transistors used in the high-voltage driver (T3 and T4; Extended Data Fig. 2a) in our system. The average power consumed by the microprocessor is only $20 \mathrm{~mW}$, and most of the power is supplied to the actuation system. The square-wave actuation signal was $115 \mathrm{~Hz}$, which was matched with the resonant frequency of the flapping-wing actuators to maximize the wing stroke. Finally, the square waves are sent to the flapping-wing actuators. The d.c.-d.c. converter efficiency was greater than $80 \%$ over a wide range of output power with a maximum efficiency of $83.1 \%$, as shown in Fig. 2 c.

Figure $2 \mathrm{~d}$ shows the output voltage for the continuous driving of the electronics; the electronics successfully produced a high-voltage actuation signal without thermal runaway. The maximum output power of the power-receiving circuit (antenna and rectifiers) is 
a

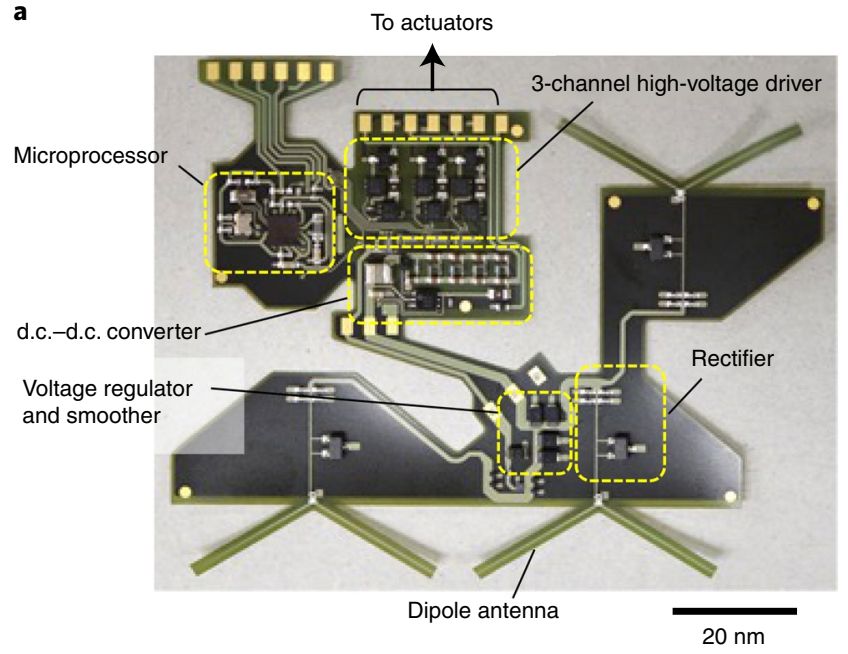

C

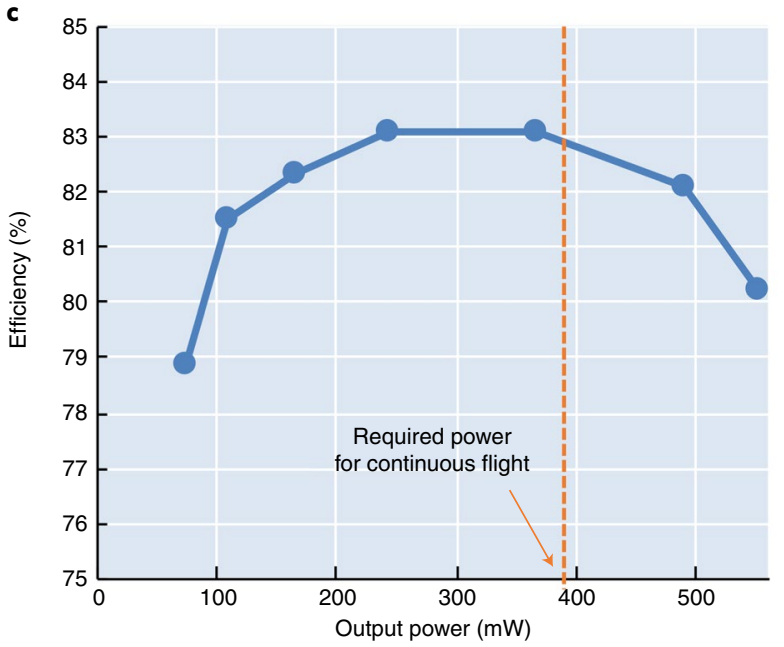

b

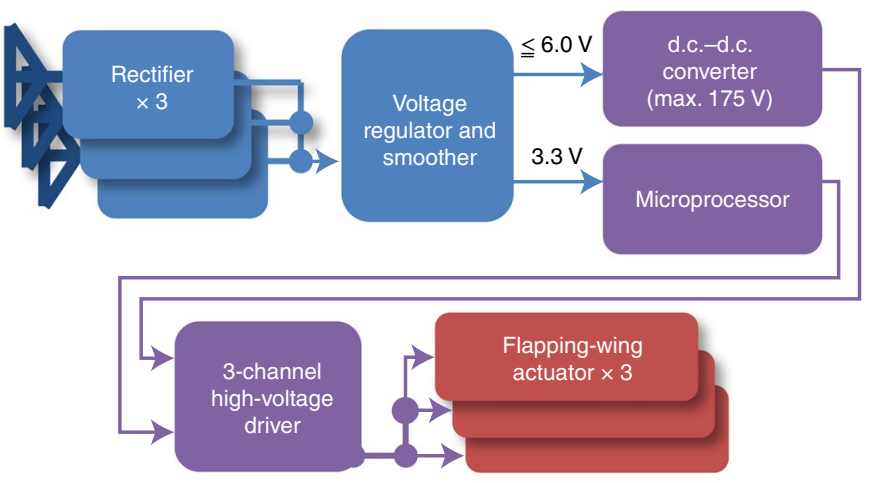

d

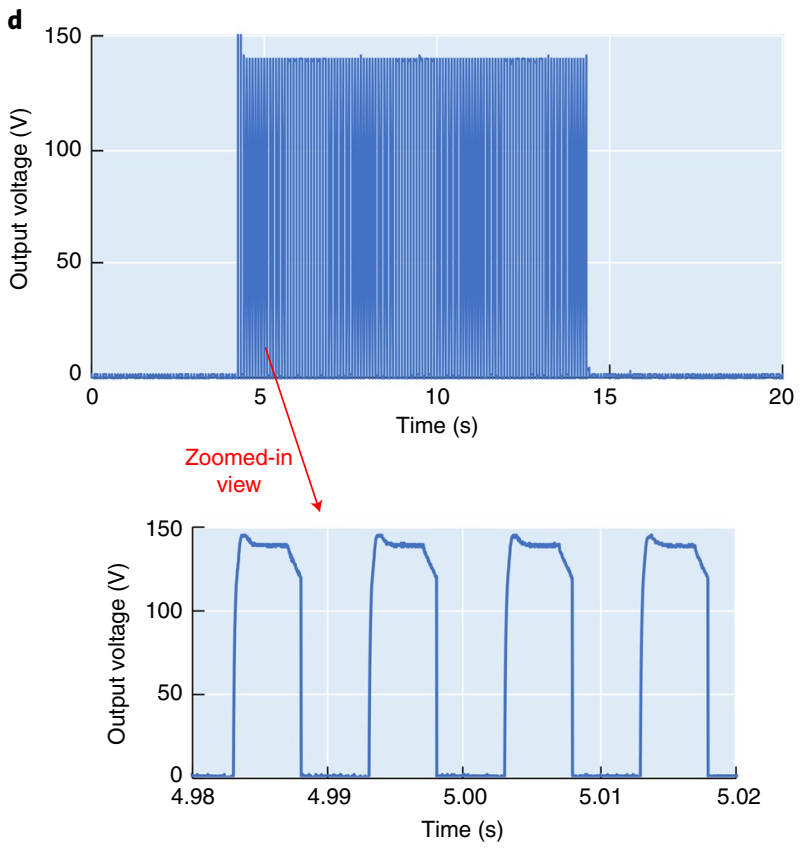

Fig. 2 | Electronics and power conversion system. a, Photograph of the fabricated MAV circuit. b, System overview. c, Efficiency of the d.c.-d.c. converter. d, Continuous drive demonstration by the RF power supply.

$1.2 \mathrm{~W}$, which is designed to meet the required power at the d.c.-d.c. converter start up. The total weight of the power receiver was $245 \mathrm{mg}$, and the power density was $4,900 \mathrm{~W} \mathrm{~kg}^{-1}$. This is much higher than off-the-shelf lithium polymer batteries, for example, the mass and power density of a GM300910 battery (PowerStream Technology) are $330 \mathrm{mg}$ and $897 \mathrm{~W} \mathrm{~kg}^{-1}$, respectively, and those of GEB201212C (PowerStream Technology) are $500 \mathrm{mg}$ and $1,200 \mathrm{~W} \mathrm{~kg}^{-1}$, respectively.

\section{Flapping-wing actuator and its performance}

The actuation mechanism is another key performance factor for MAVs. The developed vehicle employs a very simple 'direct-drive' mechanism for wing actuation, as shown in Fig. 3a. The 32.40-mm-long biomimetic wing is directly connected to a $16.50-\mathrm{mm}$-long piezoelectric bending actuator composed of a $0.10-\mathrm{mm}$-thick piezoelectric layer laminated to a $0.13-\mathrm{mm}$-thick titanium elastic plate. The bending actuator was designed as a trapezoid with the top and bottom sides measuring 4.4 and $8.5 \mathrm{~mm}$, respectively, to avoid stress concentration. Owing to the high piezoelectric coefficient of single-crystalline $\mathrm{Pb}\left(\mathrm{In}_{1 / 2} \mathrm{Nb}_{1 / 2}\right) \mathrm{O}_{3}-\mathrm{Pb}\left(\mathrm{Mg}_{1 / 3} \mathrm{Nb}_{2 / 3}\right) \mathrm{O}_{3}-\mathrm{PbTiO}_{3}$ (TRS Technologies) $^{39}$ used in this study (its effective piezoelectric coefficient $d_{32}$ is $-1,156 \mathrm{pm} \mathrm{V}^{-1}$ (ref. ${ }^{39}$ ), which is two times higher than that of typical commercial piezoelectric ceramics under large electric field $s^{40}$ ), large flapping motions are possible without the need for a complex transmission mechanism to amplify the motion if the strain during actuation is sufficiently smaller than the fracture strain of the piezoelectric material.

In our previous design ${ }^{15}$, we achieved 312 mgf of lift (sinusoidal drive with a peak-to-peak voltage of $100 \mathrm{~V}$ ) per actuator with a mass of $252 \mathrm{mg}$. To obtain sufficient payload capacity even with the installed electronics, we have improved the lift by reducing the vibration energy loss of the paired-wing configuration, and we have reduced the mass without reducing the lift by downsizing the actuators; this redesign was implemented in a previous report ${ }^{38}$, and the approach of the redesign is described in Supplementary Section 4 . The lift force and power consumption can be controlled by the duty ratio ${ }^{18,41,42}$. 
a
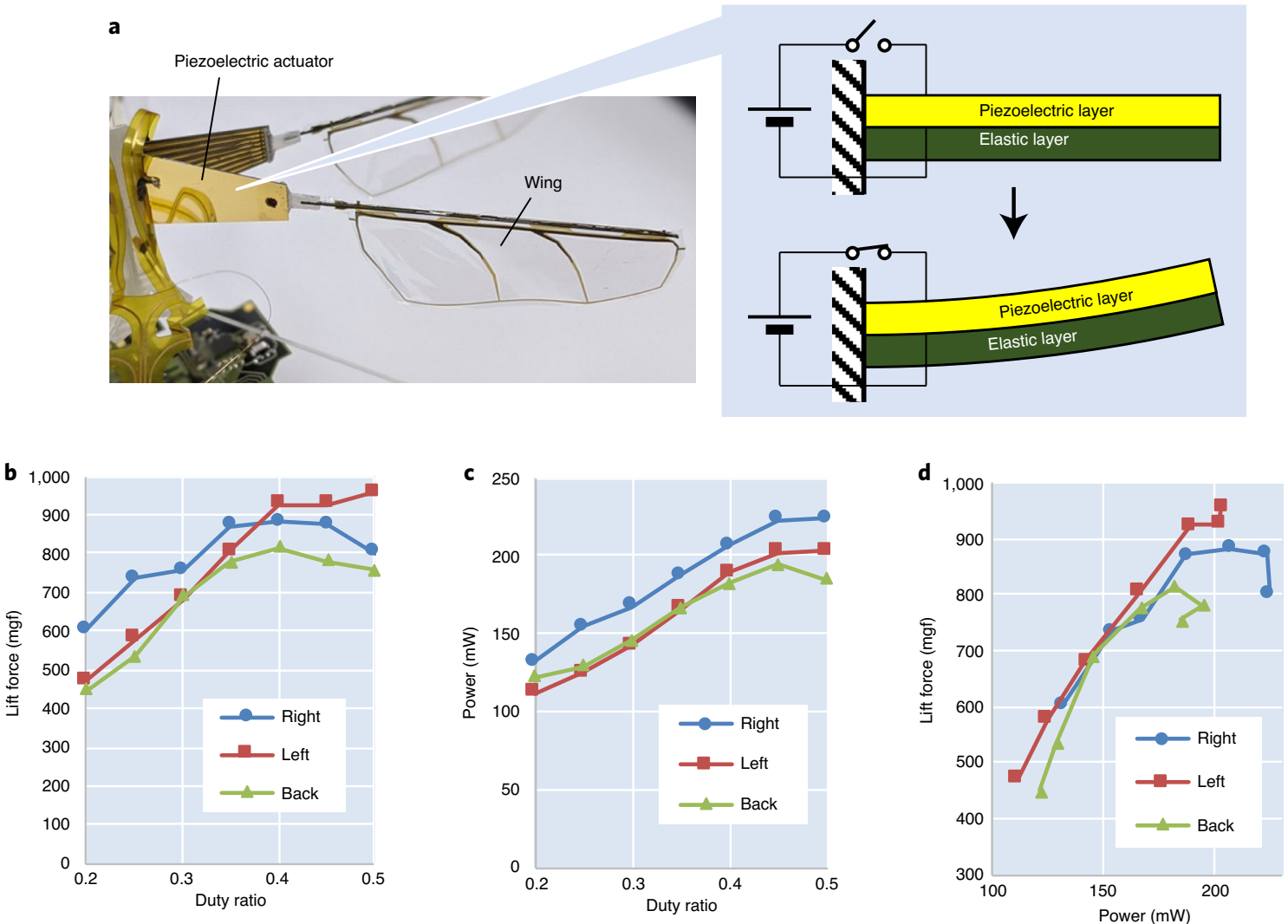

Fig. 3 | Overview and performance of flapping-wing actuator. a, Photograph of the flapping wing and actuation principle (inset shows the schematic). b, Duty ratio versus average lift force. c, Duty ratio versus consumed power. $\mathbf{d}$, Consumed power versus lift force.

Figure $3 b, c$ shows the performance of the fabricated paired actuator with a square-wave driving signal having a voltage amplitude of $140 \mathrm{~V}$ and frequency of $115 \mathrm{~Hz}$. Here the driving signal was generated by a combination of high-voltage driver circuit and d.c. voltage source (PMX350-0.2A, Kikusui Electronics). Thus, the consumed power includes the loss in the high-voltage driver, which is expected to be less than $1 \mathrm{~mW}$ (mainly due to the leakage current of the n-type metal-oxide-semiconductor field-effect transistor (nMOSFET); T3 and T4 in Extended Data Fig. 2a), which is much smaller than the handling energy. The average maximum lift force per paired actuator is $880 \mathrm{mgf}$, allowing the MAV to take off; $600 \mathrm{mgf}$ per paired actuator is required to launch the MAV because its total weight is $1,810 \mathrm{mg}$. From Fig. 3d, the required power per paired actuator is approximately $130 \mathrm{~mW}$, and the total power consumed by the actuators is estimated to be $390 \mathrm{~mW}$. The efficiency of the d.c.-d.c. converter at an output power of $390 \mathrm{~mW}$ required for continuous flight was $83 \%$ (Fig. 2c). Considering this efficiency in addition to the power consumed by the microprocessor and voltage regulator $(20-36 \mathrm{~mW})$, the total power consumption for continuous flight is approximately $500 \mathrm{~mW}$. The lift-to-power efficiency including the electronics (namely, d.c.-d.c. converter, high-voltage driver and microprocessor) was 3.6-3.7 $\mathrm{gfW}^{-1}$. This value is higher than that reported for previous insect-scale flapping-wing MAVs (FWMAVs); for example, 2.4-3.0 gf W$^{-1}$ was reported in an earlier study ${ }^{24}$. Considering the efficiency of the RF receiver, a received electromagnetic-wave power of approximately $500 \mathrm{~mW} / 67 \% \cong 750 \mathrm{~mW}$ was required for continuous flight (the power flow diagram for continuous flight is shown in Extended Data Fig. 4).

The power consumption of the piezoelectric actuator includes the reactive power due to charging/discharging of the capacitive component. The capacitance $C$ of the paired actuators is approximately
$50 \mathrm{nF}$, and the reactive power is estimated to be $C V^{2} f=113 \mathrm{~mW}$ with an ideal square-wave drive at $140 \mathrm{~V}$ (peak to peak) and $50 \%$ duty ratio. The total power consumption was approximately $230 \mathrm{~mW}$ under this condition (measured with a high-voltage amplifier and a function generator for the ideal square-wave drive). Thus, the real power is about $117 \mathrm{~mW}$, and the power efficiency is estimated to be $51 \%$. Because some of the reactive power can be recovered by circuitry ${ }^{18}$, there is room for future improvement in the efficiency of this system.

The power-to-weight efficiency of the MAV was approximately $400 \mathrm{~mW} \mathrm{~g}^{-1}$ (including the rectifier efficiency). Therefore, the actuation system of the developed vehicle is as efficient as the metabolic performance of natural bees ${ }^{43-45}$. This overall system efficiency also matches that of small quadcopters (for example, Aerix Quadrotor (10 g, $50 \mathrm{~mm}$ span) with an efficiency of $\sim 430 \mathrm{~mW} \mathrm{~g}^{-1}$ ).

\section{Untethered flight}

The orientation of the power-receiving antennas can be freely configured by folding them like origami. In this experiment, two of the three power-receiving antennas were laid out parallel to each other, whereas the third antenna was laid out orthogonally (Fig. 4a). The orientation of the two antennas coincided with the polarization of the transmitting antenna during standby, and the two antennas received a large amount of power for circuit start up. The orthogonal antenna allows the vehicle to receive energy even when the vehicle is rotating. A horn antenna was chosen for the transmitter; because the electromagnetic waves were transmitted at a constant power (we used $30 \mathrm{~W}$, which is the maximum power of our instruments), the power received by the vehicle depended on its distance from the transmitting antenna. This power characteristic limits the operating distance of the vehicle. 
a

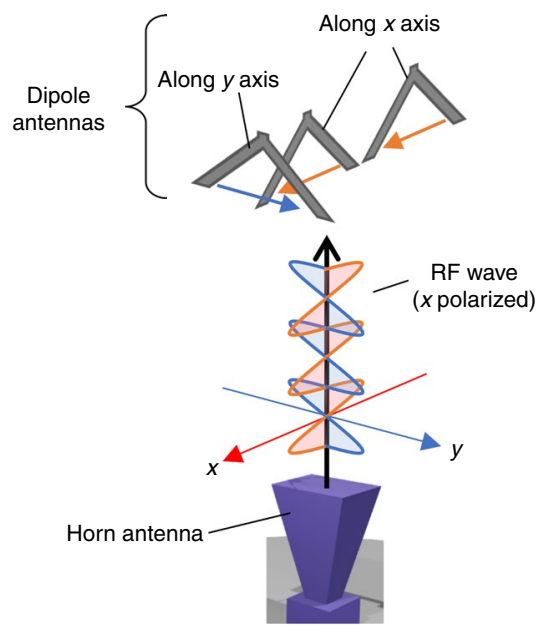

C

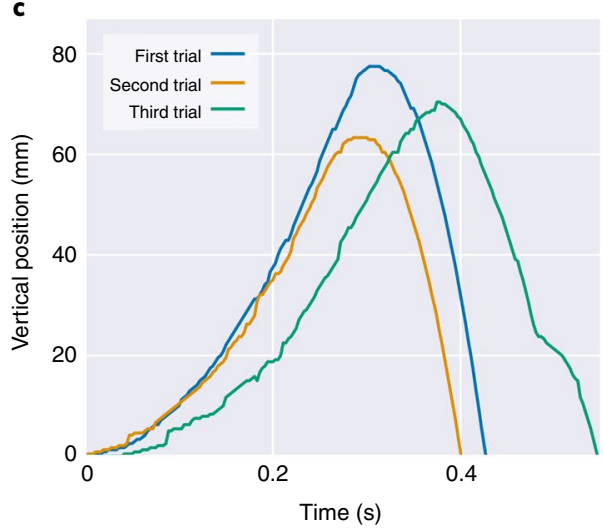

b

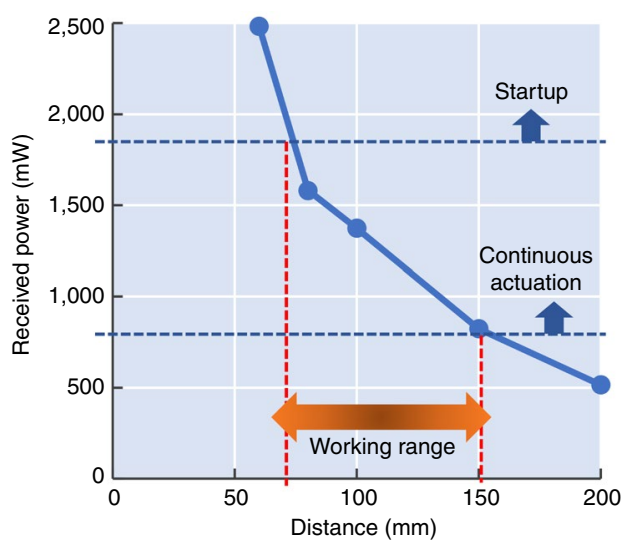

d

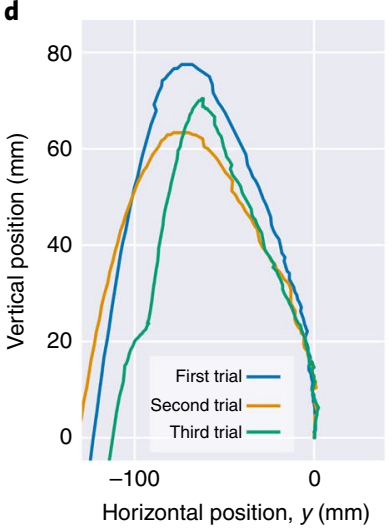

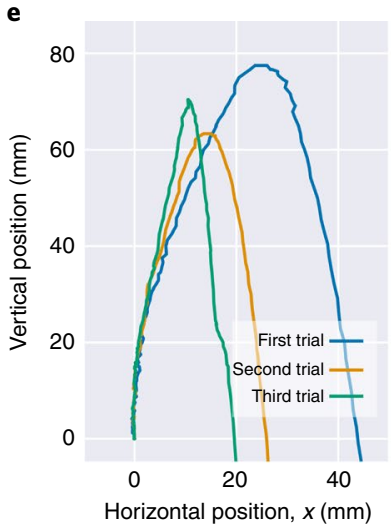

Fig. 4 | Configuration and actuation performance of wireless-powered flight. $\mathbf{a}$, Antenna layout of the aircraft. $\mathbf{b}$, Relationship between received power and distance from the transmitting antenna to the receiving horn-dipole antenna. c, Transition in height of the aircraft during flight demonstration. d,e, Flight trajectories in $y(\mathbf{d})$ and $x$ (e) directions.

The relationship between the power received at the dipole antenna and the vertical distance of the vehicle from the transmitter is shown in Fig. 4b (three-dimensional distribution of the received power is shown in Extended Data Fig. 5). The vehicle can be operated with a power that is sufficiently large for continuous actuation but not unnecessarily greater than the circuit start-up power to avoid thermal runaway. The operating vertical-distance range was $70-150 \mathrm{~mm}$ in the experimental setup. From this result, to maximize the flight distance, we determined the initial distance from the vehicle to the transmitting antenna and transmission power as $70 \mathrm{~mm}$ and $30 \mathrm{~W}$, respectively.

The trajectories of three flight trials are shown in Fig. $4 \mathrm{c}-\mathrm{e}$, where the zero of the vertical position means the initial position of the vehicles. The flight performance of the vehicle had good reproducibility; the maximum altitudes showed a deviation of $18.8 \%$. We inferred that the trajectory difference was mainly caused by the error in the initial position of the vehicle. Figure $4 \mathrm{c}$ shows the accelerations in altitude. These suggest that the flights were not just jumps, but flights with continuous thrust. The maximum altitude in this demonstration was $77.5 \mathrm{~mm}$. The altitude at which the flapping stopped is thought to have been near the inflection point of the trajectory. We calculated the inflection point by fitting the trajectory with a quadratic curve (Extended Data Fig. 6, top). As a result, it was estimated that the actuator stopped at an altitude of $58-66 \mathrm{~mm}$.

Based on the coordinates at the inflection point and the received power distribution, the received power at the inflection was estimated to be approximately $600 \mathrm{~mW}$ (Extended Data Fig. 6, bottom).
This coincides with the estimated power required for continuous flight $(\sim 750 \mathrm{~mW})$. This is further evidence that the system was continuously powered by the RF supply. The excess lift, excluding the self-weight, during the ascent estimated from the above fitting results was 220-340 mgf. Note that we did not implement closed-loop attitude control in this experiment, because this vehicle was not equipped with any attitude estimation sensor. Instead, we carefully tuned the duty ratios of the actuation signals based on the duty ratio-lift force data (Fig. 3b) to balance the vehicle and achieve level flight; we employed duty ratios of $0.30,0.47$ and 0.49 for the right, left and back actuators, respectively. As shown in Fig. 3b, the total lift force is estimated to be about $2.4 \mathrm{gf}$ at the duty ratios setting. On the other hand, the total lift force was estimated to be $\sim 2.0-2.2 \mathrm{gf}$ from the excess lift force calculated from the trajectory described above. We expect that this reduction in lift force is mainly due to the fixed state of the actuator. For the measurement shown in Fig. 3, the paired actuators were rigidly fixed. In the case of the vehicle floating in air, the lift force may have decreased due to the vibration of the entire vehicle. Another possible cause is the tilt of the vehicle. The vehicle was tilted by up to $20^{\circ}$ during flight, potentially causing a lift loss of several percentages in the vertical direction.

The most important improvements that need to be made in the present system should be the extension of the operating range (distance) and the reduction in transmission power. To extend the operating range, a system to adjust the power supply according to the distance of the aircraft is needed. The RF power is inversely proportional to the square of the transmitter-receiver distance and 
proportional to the transmitting power. Thus, if the power is increased, the distance that can be travelled should increase in proportion to the square root of the transmitting power. However, excessive reception energy leads to failure due to overheating of the circuit; therefore, the distance that can be approached by the transmitting antenna also increases. To reduce the transmitting power, a system that focuses on the electromagnetic waves, such as phased arrays, could be promising. Either way, a system that measures the current position of the aircraft and feeds it back to the transmitter will be necessary.

\section{Conclusions}

We have shown that RF power receivers can be used as sustainable power sources for insect-scale robots. Our circuit design consists of three dipole antennas/rectifiers to avoid heat concentration, and the high power density was created using a lightweight RF power receiver. We integrated it to an $\mathrm{FWMAV}^{38}$ with a high lift-to-power efficiency. Key performance metrics, namely, power density and total weight of the aircraft, exceed those of existing designs. The power density of our RF power receiver is four times higher than that for off-the-shelf lithium polymer batteries. This is also 2.1 times the power density provided by the solar panel $\left(2,300 \mathrm{~W} \mathrm{~kg}^{-1}\right)$ used for untethered flight of an insect-scale FWMAV reported previously ${ }^{24}$. With regard to the total weight, previously reported motor-driven multirotor MAVs with RF power transmission weigh several tens of grams (for example, $51 \mathrm{~g}\left(\right.$ ref. ${ }^{30}$ ) and $85 \mathrm{~g}$ ); our vehicle is an order of magnitude lighter than these examples.

We believe a fully functional untethered MAV with insect-scale mass can be realized by optimizing the vehicle design and adding new features. These include integrating a flight control system in the FWMAV and using a beam-focusing/targeting system for the power transmitter. Beam focusing is also important from a regulatory point of view because the output power of the transmitting antenna is limited below a certain level. For example, according to the Federal Communications Commission (USA) and the Ministry of Internal Affairs and Communications (Japan), the output power of indoor antennas must be less than $1 \mathrm{~W}$ (refs. ${ }^{46,47}$ ). The use of square waves has the advantage of lower peak-to-peak voltage and a simpler drive circuit compared with those required to generate a sinusoidal actuation signal. On the other hand, the steep drive may increase the risk of actuator damage. The verification of the long-term reliability of this system will be another important future step. Furthermore, a closed-loop flight control system could also be implemented and an altitude-sensing system, such as an inertial measurement unit, could be included in the electronics. An additional antenna for wireless communication is also desirable for practical applications. We note that the developed vehicle has sufficient payload capacity to carry such sensors: the maximum total lift force and payload were 2,620 and $810 \mathrm{mgf}$, respectively; the typical mass of a commercial inertial measurement unit or communication antenna (such as Bluetooth) is several tens of milligrams only.

\footnotetext{
Methods

Vehicle fabrication. The body and legs of the vehicle were fabricated from glass-fibre-reinforced plastic (GFRP) plates with a thickness of $0.38 \mathrm{~mm}$ (FS-10, ACP Composites) and GFRP rods with a diameter of $0.5 \mathrm{~mm}$ (Featherfield), respectively. The GFRP parts, flapping-wing actuators and circuit board were joined using an ultraviolet-curable adhesive (LOCTITE 4310). Enamel wire with a diameter of $70 \mu \mathrm{m}$ was used to wire the actuator to the circuit; the circuit-side and actuator-side ends were connected using a solder and conductive adhesive (CW2400, Chemtronics), respectively. In our earlier reports, we have comprehensively described the fabrication processes for the vehicle (without electronics $)^{38}$ and the wings ${ }^{48}$. The mass of the six wings, six actuators, body and three legs before assembly was $107,696,340$ and $71 \mathrm{mg}$, respectively. The weight of the assembled vehicle without electronics and wiring was $1,264 \mathrm{mg}$; $50 \mathrm{mg}$ was added by the adhesive. The image of the vehicle assembly is shown in Supplementary Section 2 .
}

Circuit. The schematic of the power-receiving and converting circuits is shown in Extended Data Fig. 1. The circuit board was manufactured by Cir-Tech (Hamamatsu). The FPC comprises resist lamination, $\mathrm{Cu}$ pattern, polyimide, and $\mathrm{Cu}$ pattern layers; their thicknesses are $20 \mu \mathrm{m}, 9 \mu \mathrm{m}$ (with $7 \mu \mathrm{m} \mathrm{Cu}$ platting), $50 \mu \mathrm{m}$ and $9 \mu \mathrm{m}$ (with $7 \mu \mathrm{m} \mathrm{Cu}$ platting), respectively. The power-receiving circuit (Extended Data Fig. 1a) consists of rectifiers, voltage-limiting diodes (D2), power storage capacitors (C2) and a voltage regulator (IC1). The voltage-limiting diode protects the d.c. - d.c. converter and limits the output voltage below $6 \mathrm{~V}$ (any input beyond this voltage is dissipated as heat loss). The power storage capacitors are used to smooth the output voltage and absorb fluctuations due to the instantaneous load power. A voltage regulator is used to supply power to the digital circuits (for example, microcontroller), and the output is $3.3 \mathrm{~V}$. We employed a combination of boost converter and charge pump for the d.c.-d.c. converter; the input voltage $(<6 \mathrm{~V})$ is boosted to a maximum of $35 \mathrm{~V}$ and then multiplied by five (Extended Data Fig. 1b): $35 \times 5=175 \mathrm{~V}$. The boost converter is controlled by XC9104D095DR-G (Torex Semiconductor) with an operation frequency of $500 \mathrm{kHz}$. The high-voltage driving circuit is based on the one developed by us in an earlier study ${ }^{18}$ (the full schematic is shown in Extended Data Fig. 2a); note that the charge recovery reported in ref. ${ }^{18}$ was not used in this experiment. This high-voltage driver is composed of a combination of bipolar transistor and nMOSFET. We selected the components among the available ones along with focusing on withstanding the voltage and mass (the breakdown of the circuit mass is summarized in Extended Data Table 2 and the complete electrical component list is shown in Extended Data Table 3). The schematic of the rectifier and the simulated performance using electronic design automation software (advanced design system, Keysight Technologies) are shown in Extended Data Fig. 1c-e. There are four types of power entering and leaving the rectifier: input power $\left(P_{\mathrm{I}}\right.$; Extended Data Fig. 1c), output power $\left(P_{\mathrm{O}}\right.$; Extended Data Fig. 1c), internal losses (diodes and wires) and reflections back to the input port. Extended Data Fig. 1d shows the internal loss against the input power at a constant $P_{\mathrm{O}}$. When $P_{\mathrm{I}}$ increases, the internal loss increases almost proportionally. Since the increase in internal losses leads to the risk of thermal runaway, it is important to appropriately adjust $P_{\mathrm{I}}$. As shown in Extended Data Fig. 1e, the maximum efficiency of the rectifier $\left(P_{\mathrm{O}} / P_{\mathrm{I}}\right)$ was approximately $67 \%$ with an optimized input power at $P_{\mathrm{O}}=500 \mathrm{~mW}$, which is required for continuous flight. This efficiency is comparable to larger rectifiers ${ }^{49,50}$.

The d.c. - d.c. conversion efficiency. To determine the d.c. - d.c. conversion efficiency (Fig. 2c), the circuit was powered by a d.c. voltage source (E3631A, Agilent Technologies) and the input and output powers were evaluated by power analysers (PA1000, Tektronix). The input voltage was set to $3.7 \mathrm{~V}$ in this evaluation. The output voltage was set to $140 \mathrm{~V}$. Using the fabricated high-voltage driver, we drive a pseudo-load with a duty ratio of $50 \%$. Here we used an equivalent pseudo-load composed of resistor-capacitor parallel circuits (resistance, $R=133 \mathrm{k} \Omega$; capacitance, $C=50 \mathrm{nF}$ ) instead of real actuators to prevent the breakage of the actuator caused by excessive drive power. The electrical characteristics of piezoelectric actuators can be modelled as a parallel circuit of a capacitor and a resistor-inductor-capacitor (RLC) series in a simplistic form ${ }^{51,52}$. The capacitance and resistance values are estimated based on the power consumption characteristics around the resonance frequency (the fitting method and result are shown in Extended Data Fig. 7). With a voltage amplitude of $140 \mathrm{~V}$ and a duty ratio of $50 \%$, the power consumptions of the pseudo-load circuit and real actuator were 204 and $186 \mathrm{~mW}$, respectively (the error was $8.6 \%$ ). We adjusted the output power by changing the resistance and capacitance.

Continuous driving test of circuit. In the continuous driving test (Fig. 2d), we used the equivalent pseudo-load employed in the measurement of the d.c.-d.c. converter. The RF wave was generated by a system composed of a programmable voltage source (2461, Keithley), 5.8 GHz RF amplifier (OSGM50-5.7G5.9G, Plasma Applications) and horn antenna (1424-15, Flann Microwave). The output RF power was $30 \mathrm{~W}$. The output signal was recorded by an oscilloscope (DPO4034B, Tektronix)

Actuator performance. The driving signal was generated by a combination of the high-voltage driver circuit and a d.c. voltage source (PMX350-0.2A, Kikusui Electronics). Here the input voltage was set to $140 \mathrm{~V}$. The control signal for the high-voltage driver was generated by the microprocessor circuit, where the voltage and frequency of the control signal were set to $3.3 \mathrm{~V}$ and $115 \mathrm{~Hz}$, respectively. The lift force was measured by a precision electrical balance (HR-100A, A\&D Company); the experimental layout is the same as reported earlier ${ }^{18}$.

Distance dependency of received power by dipole antenna. The RF wave was generated by the same instruments as used in the continuous driving test. The received power was measured by a combination of our fabricated FPC dipole antenna and an attenuator $(-30 \mathrm{~dB})(\mathrm{E} 9301 \mathrm{~B}$, Keysight Technologies) in series with a power sensor (E9304A or N1913A, Keysight Technologies).

Flight demonstration. The RF wave was generated by the same instruments that were used in the continuous driving test. The output RF power was $30 \mathrm{~W}$. The 
flight trajectory was captured by a motion-tracking system (Prime ${ }^{\mathrm{x}} 41$, OptiTrack) and its image was recorded by a colour camera included with the system. The vehicle was placed on a $160 \mathrm{~mm}$ square acrylic plate above the transmitting horn antenna with an initial distance of $70 \mathrm{~mm}$ from the transmitting antenna to the receiving dipole antenna.

\section{Data availability}

The data that support the findings of this study are available from the corresponding author upon reasonable request.

Received: 21 March 2021; Accepted: 5 October 2021; Published online: 25 November 2021

\section{References}

1. Rossi, M. et al. Gas-drone: portable gas sensing system on UAVs for gas leakage localization. In Sensors, 2014 IEEE 1431-1434 (IEEE 2014).

2. Alvissalim, M. S. et al. Swarm quadrotor robots for telecommunication network coverage area expansion in disaster area. In 2012 Proc. SICE Annual Conference (SICE) 2256-2261 (IEEE, 2012).

3. Naqvi, S. A. R., Hassan, S. A., Pervaiz, H. \& Ni, Q. Drone-aided communication as a key enabler for $5 \mathrm{G}$ and resilient public safety networks. IEEE Commun. Mag. 56, 36-42 (2018).

4. Kim, S. J., Jeong, Y., Park, S., Ryu K. \& Oh, G. A survey of drone use for entertainment and AVR (augmented and virtual reality). In Augmented Reality and Virtual Reality. Progress in IS (eds. Jung, T. \& tom Dieck, M.) 339-352 (Springer, 2017).

5. Bi, Y., Lan, M., Li, J., Lai, S. \& Chen, B. M. A lightweight autonomous MAV for indoor search and rescue. Asian J. Control. 21, 1732-1744 (2019).

6. Rabta, B., Wankmüller, C. \& Reiner, G. A drone fleet model for last-mile distribution in disaster relief operations. Int. J. Disaster Risk Reduct. 28, 107-112 (2018).

7. United States Department of Transportation. Unmanned Aircraft Systems (UAS) https://www.faa.gov/uas/ (2020).

8. Niekawa, Shun. Japan to expand drone rules so smaller, lighter models covered. The Asahi Shimbun. http://www.asahi.com/ajw/articles/13997174 (2020).

9. Steltz, E., Seeman, M., Avadhanula, S. \& Fearing, R. S. Power electrics design choice for piezoelectric microrobots. In 2006 IEEE/RSJ International Conference on Intelligent Robots and Systems 1322-1328 (IEEE, 2006).

10. Karpelson, M., Wei, G.-Y. \& Wood, R. J. A review of actuation and power electronics options for flapping-wing robotic insects. In 2008 IEEE International Conference on Robotics and Automation 779-786 (IEEE, 2008).

11. Wood, R. J. Liftoff of a 60mg flapping-wing MAV. In 2007 IEEE/RSJ International Conference on Intelligent Robots and Systems 1889-1894 (IEEE, 2007)

12. Steltz, E., Avadhanula, S. \& Fearing, R. S. High lift force with $275 \mathrm{~Hz}$ wing beat in MFI. In 2007 IEEE/RSJ International Conference on Intelligent Robots and Systems 3987-3992 (IEEE, 2007).

13. Anderson, M. L. Design and Control of Flapping Wing Micro Air Vehicles. PhD thesis, Air Force Institute of Technology Wright-Patterson Air Force Base, $\mathrm{OH}$, USA (2011).

14. Hines, L. Design and Control of a Flapping Flight Micro Aerial Vehicle. $\mathrm{PhD}$ thesis, Carnegie Mellon Univ. (2014).

15. Ozaki, T. \& Hamaguchi, K. Bioinspired flapping-wing robot with direct-driven piezoelectric actuation and its takeoff demonstration. IEEE Robot. Autom. Lett. 3, 4217-4224 (2018).

16. Campolo, D., Sitti, M. \& Fearing, R. S. Efficient charge recovery method for driving piezoelectric actuators with quasi-square waves. IEEE Trans. Ultrason., Ferroelectr., Freq. Control. 50, 1-10 (2003).

17. Karpelson, M., Wei, G.-Y. \& Wood, R. J. Driving high voltage piezoelectric actuators in microrobotic applications. Sens. Actuator A Phys. 176, 78-89 (2012)

18. Ozaki, T. \& Ohta, N. Power-efficient driver circuit for piezo electric actuator with passive charge recovery. Energies 13, 2866 (2020).

19. Helbling, E. F. \& Wood, R. J. A review of propulsion, power, and control architectures for insect-scale flapping-wing vehicles. ASME. Appl. Mech. Rev. 70, 010801 (2018).

20. de Croon, G. C. H. E., Perçin, M., Remes, B. D. W., Ruijsink, R. \& Wagter, C. D. The DelFly (Springer, 2016)

21. Karásek, M., Muijres, F. T., Wagter, C. D., Remes, B. D. W. \& Croon, G. C. H. E. A tailless aerial robotic flapper reveals that flies use torque coupling in rapid banked turns. Science 361, 1089-1094 (2018).

22. Rosen, M. H., le Pivain, G., Sahai, R., Jafferis, N. T. \& Wood, R. J. Development of a $3.2 \mathrm{~g}$ untethered flapping-wing platform for flight energetics and control experiments. In 2016 IEEE International Conference on Robotics and Automation (ICRA) 3227-3233 (IEEE, 2016).

23. James, J., Iyer. V., Chukewad. Y., Gollakota, S. \& Fuller. S. B. Liftoff of a $190 \mathrm{mg}$ laser-powered aerial vehicle: the lightest wireless robot to fly. In 2018 IEEE International Conference on Robotics and Automation (ICRA) 3587-3594 (IEEE, 2018)
24. Jafferis, N. T., Helbling, E. F., Karpleson, M. \& Wood, R. J. Untethered flight of an insect-sized flapping-wing microscale aerial vehicle. Nature $\mathbf{5 7 0}$, 491-495 (2019).

25. Guo, H., Li, J., Wang, X. \& Shang, J. Research review on the development of liquid crystal optical phased array device on the application of free space laser communication. In Proc. SPIE 10841, 9th International Symposium on Advanced Optical Manufacturing and Testing Technologies: Meta-Surface-Wave and Planar Optics 108410C (SPIE, 2018).

26. He, J., Dong, T. \& Xu, Y. Review of photonic integrated optical phased arrays for space optical communication. IEEE Access 8, 188284-188298 (2020).

27. Hsieh, L. H. et al. Development of a retrodirective wireless microwave power transmission system. In IEEE Antennas and Propagation Society International Symposium 393-396 (IEEE, 2003).

28. Homma, Y. et al. New phased array and rectenna array systems for microwave power transmission research. In 2011 IEEE MTT-S International Microwave Workshop Series on Innovative Wireless Power Transmission: Technologies, Systems, and Applications 59-62 (IEEE, 2011).

29. Shinohara, N. Beam control technologies with a high-efficiency phased array for microwave power transmission in Japan. Proc. IEEE 101, 1448-1463 (2013). 30. Aldhaher, S., Mitcheson, P. D., Arteaga, J. M., Kkelis, G. \& Yates, D. C. Light-weight wireless power transfer for mid-air charging of drones. In 2017 11th European Conference on Antennas and Propagation (EUCAP) 336-340 (IEEE, 2017).

31. Plaizier, G. M. et al. Design, modeling, and analysis of inductive resonant coupling wireless power transfer for micro aerial vehicles (MAVs). In 2018 IEEE International Conference on Robotics and Automation (ICRA) 6104-6109 (IEEE, 2018).

32. Arteaga, J. M. et al. Dynamic capabilities of multi-MHz inductive power transfer systems demonstrated with batteryless drones. IEEE Trans. Power Electron. 34, 5093-5104 (2019).

33. Jawad, A. M. et al. Wireless power transfer with magnetic resonator coupling and sleep/active strategy for a drone charging station in smart agriculture. IEEE Access 7, 139839-139851 (2019).

34. Wu, S., Cai, C., Jiang, L., Li, J. \& Yang, S. Unmanned aerial vehicle wireless charging system with orthogonal magnetic structure and position correction aid device. IEEE Trans. Power Electron. 36, 7564-7575 (2021).

35 . Karpelson, M. et al. A wirelessly powered, biologically inspired ambulatory microrobot. In 2014 IEEE International Conference on Robotics and Automation (ICRA) 2384-2391 (IEEE, 2014).

36. Boyvat, M., Koh, J.-S. \& Wood, R. J. Addressable wireless actuation for multijoint folding robots and devices. Sci. Robot. 2, eaan1544 (2017).

37. Phan, H. V. \& Park, H. C. Insect-inspired, tailless, hover-capable flapping-wing robots: recent progress, challenges, and future directions. Prog. Aerosp. Sci. 111, 100573 (2019).

38. Ozaki, T. \& Hamaguchi, K. Improved lift force of a resonant-driven flapping-wing micro aerial vehicle by suppressing wing-body and wing-wing vibration coupling. Extrem. Mech. Lett. 40, 100867 (2020).

39. Sun, E., Zhang, S., Luo, J., Shrout, T. R. \& Cao, W. Elastic, dielectric, and piezoelectric constants $\mathrm{Pb}\left(\mathrm{In}_{1 / 2} \mathrm{Nb}_{1 / 2}\right) \mathrm{O}_{3}-\mathrm{Pb}\left(\mathrm{Mg}_{1 / 3} \mathrm{Nb}_{2 / 3}\right) \mathrm{O}_{3}-\mathrm{PbTiO}_{3}$ single crystal poled along [011]. Appl. Phys. Lett. 97, 032902 (2010).

40. Bruno, B. P., Fahmy, A. R., Stürmer, M., Wallrabe, U. \& Wapler, M. C. Properties of piezoceramic materials in high electric field actuator applications. Smart Mater. Struct. 28, 015029 (2019).

41. Sitti, M., Campolo, D., Yan, J. \& Fearing R. S. Development of PZT and PZN-PT based unimorph actuators for micromechanical flapping mechanisms. In Proc. 2001 ICRA. IEEE International Conference on Robotics and Automation 3839-3846 (IEEE, 2001).

42. Peng, Y. et al. A piezo-driven flapping wing mechanism for micro air vehicles. Microsyst. Technol. 23, 967-973 (2017).

43. Nachtigall, W., Rothe, U., Feller, P. \& Jungmann, R. Flight of the honey bee. J. Comp. Physiol. B 158, 729-737 (1989).

44. Nachtigall, W., Hanauer-Thieser, U. \& Mörz, M. Flight of the honey bee VII: metabolic power versus flight speed relation. J. Comp. Physiol. B $\mathbf{1 6 5}$, 484-489 (1995).

45. Schmolz, E., Brüders, N., Schricker, B. \& Lamprecht, I. Direct calorimetric measurement of heat production rates in flying hornets (Vespa crabro; Hymenoptera). Thermochim. Acta 328, 3-8 (1999).

46. National Archives and Records Administration \& US Government Publishing Office. Code of Federal Regulations https://ecfr.federalregister.gov/current/ title-47/chapter-I/subchapter-A/part-15 (2021).

47. Telecommunications Bureau of the Ministry of Internal Affairs and Communications, Government of Japan. The Radio Use Web Site https://www. tele.soumu.go.jp/j/sys/others/wlan_outdoor/index.htm (2020).

48. Ozaki, T. \& Hamaguchi, K. Batch fabrication process of biomimetic wing with high flexibility of stiffness design for flapping-wing micro aerial vehicles. MethodsX 7, 101121 (2020).

49. Mabrouki, A., Latrach, M. \& Z. Sayegh. Design and experiment of RF rectifiers for wireless power transmission. In 2013 13th Mediterranean Microwave Symposium (MMS) 1-4 (IEEE, 2013). 
50. Song, C., Huang, Y., Zhou, J. \& Carter, P. Recent advances in broadband rectennas for wireless power transfer and ambient RF energy harvesting. In 2017 11th European Conference on Antennas and Propagation (EUCAP) 341-345 (2017).

51. Goldfarb, M. \& Celanovic, N. Modeling piezoelectric stack actuators for control of micromanipulation. IEEE Control Syst. Mag. 17, 69-79 (1997).

52. Harter, J. H. Essentials of Electric Circuits 670-676 (Reston, 1982).

\section{Acknowledgements}

We thank M. Ishida and T. Watanabe for assistance in operating the RF instruments and in programming and running the design software.

\section{Author contributions}

T.O. designed, fabricated and evaluated the mechanical part of the vehicle. He also assembled the whole vehicle. N.O. designed, fabricated and evaluated the electronics of the vehicle. T.O. and N.O. conducted the flight demonstration experiment. T.J. and K.H. analysed the system performance. T.O. wrote the paper. All the authors provided critical feedback.

\section{Competing interests}

The authors declare no competing interests.

\section{Additional information}

Extended data is available for this paper at https://doi.org/10.1038/s41928-021-00669-8. Supplementary information The online version contains supplementary material available at https://doi.org/10.1038/s41928-021-00669-8.

Correspondence and requests for materials should be addressed to Takashi Ozaki.

Peer review information Nature Electronics thanks Elizabeth Helbling and Noah Jafferis for their contribution to the peer review of this work.

Reprints and permissions information is available at www.nature.com/reprints.

Publisher's note Springer Nature remains neutral with regard to jurisdictional claims in published maps and institutional affiliations.

(c) (i) Open Access This article is licensed under a Creative Commons

Attribution 4.0 International License, which permits use, sharing, adaptation, distribution and reproduction in any medium or format, as long as you give appropriate credit to the original author(s) and the source, provide a link to the Creative Commons license, and indicate if changes were made. The images or other third party material in this article are included in the article's Creative Commons license, unless indicated otherwise in a credit line to the material. If material is not included in the article's Creative Commons license and your intended use is not permitted by statutory regulation or exceeds the permitted use, you will need to obtain permission directly from the copyright holder. To view a copy of this license, visit http://creativecommons. org/licenses/by/4.0/.

(c) The Author(s) 2021 
a Rectifier
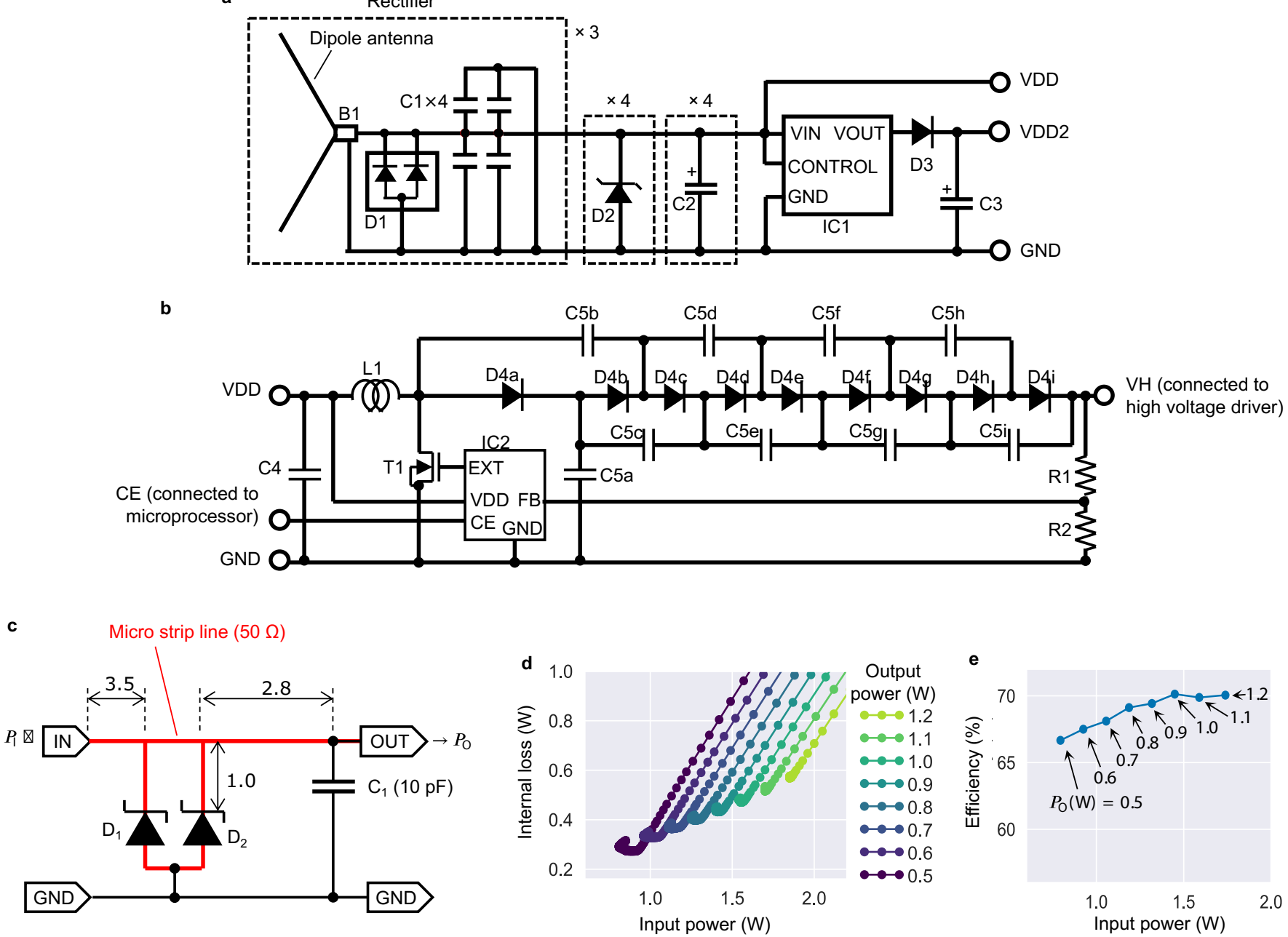

Extended Data Fig. 1 | Schematics of power-receiving and conversion circuit. Schematics of power-receiving and conversion circuit. a, Schematics of RF power receiver. b, Schematics of high-voltage DC-DC converter. c, Schematic of rectifier circuit. d, Input power-loss characteristics. e, Input powerefficiency characteristics. 


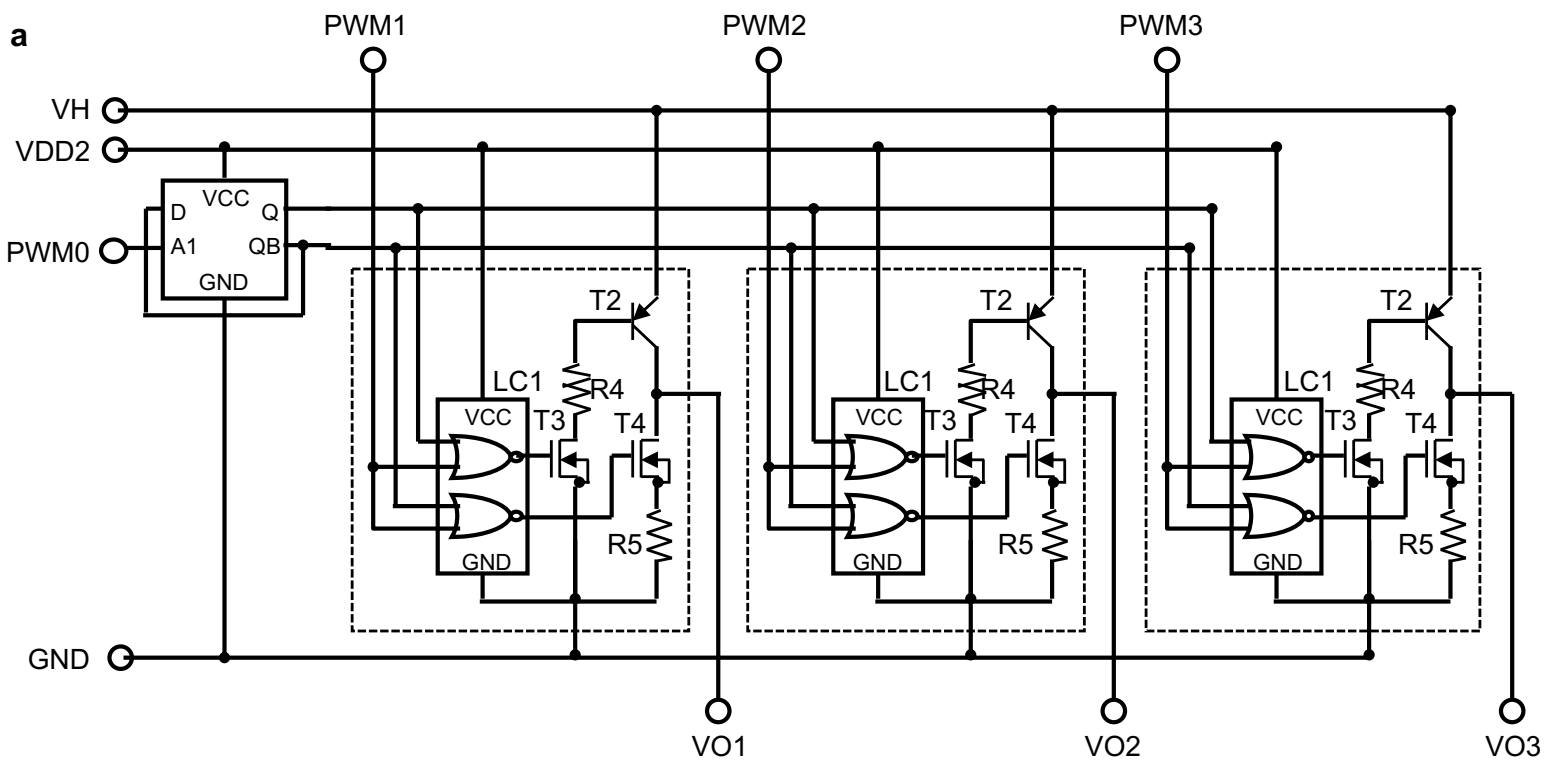

b

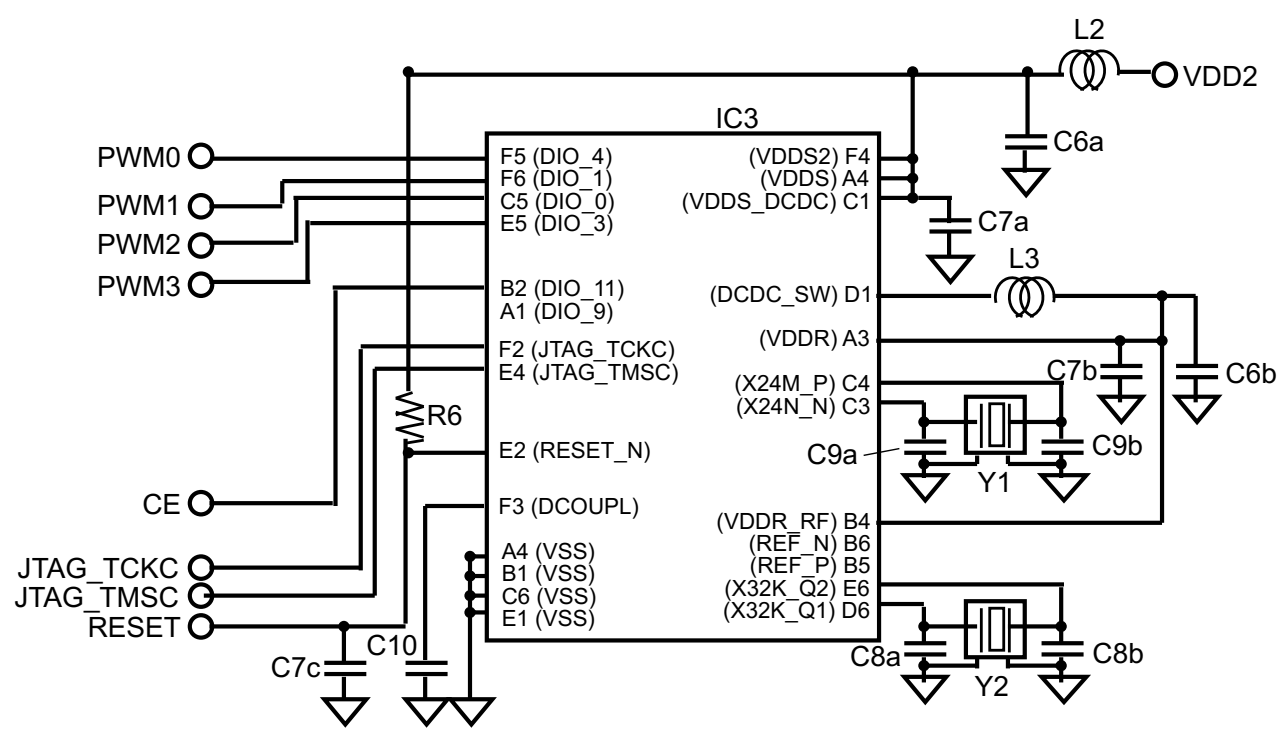

c

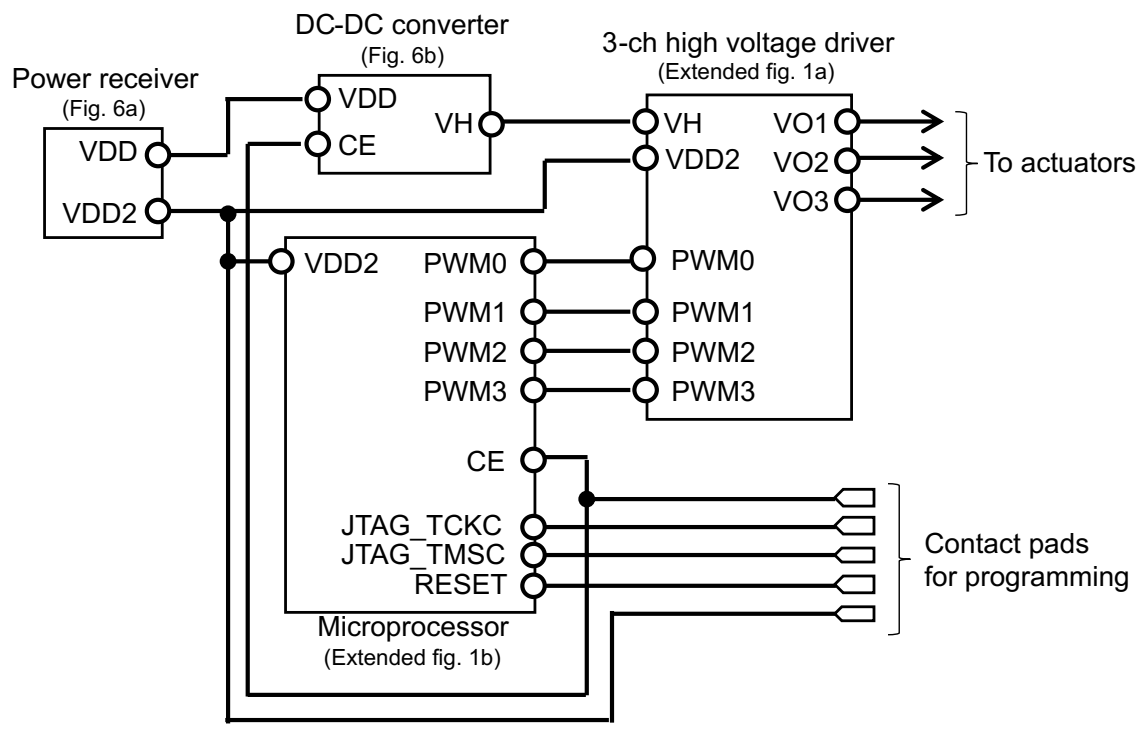

Extended Data Fig. 2 | Schematics of high voltage driver and microprocessor. Schematics of high voltage driver and microprocessor. (a), high voltage driver (b), microprocessor and its peripheral components, (c) connection diagram of subcircuits. 


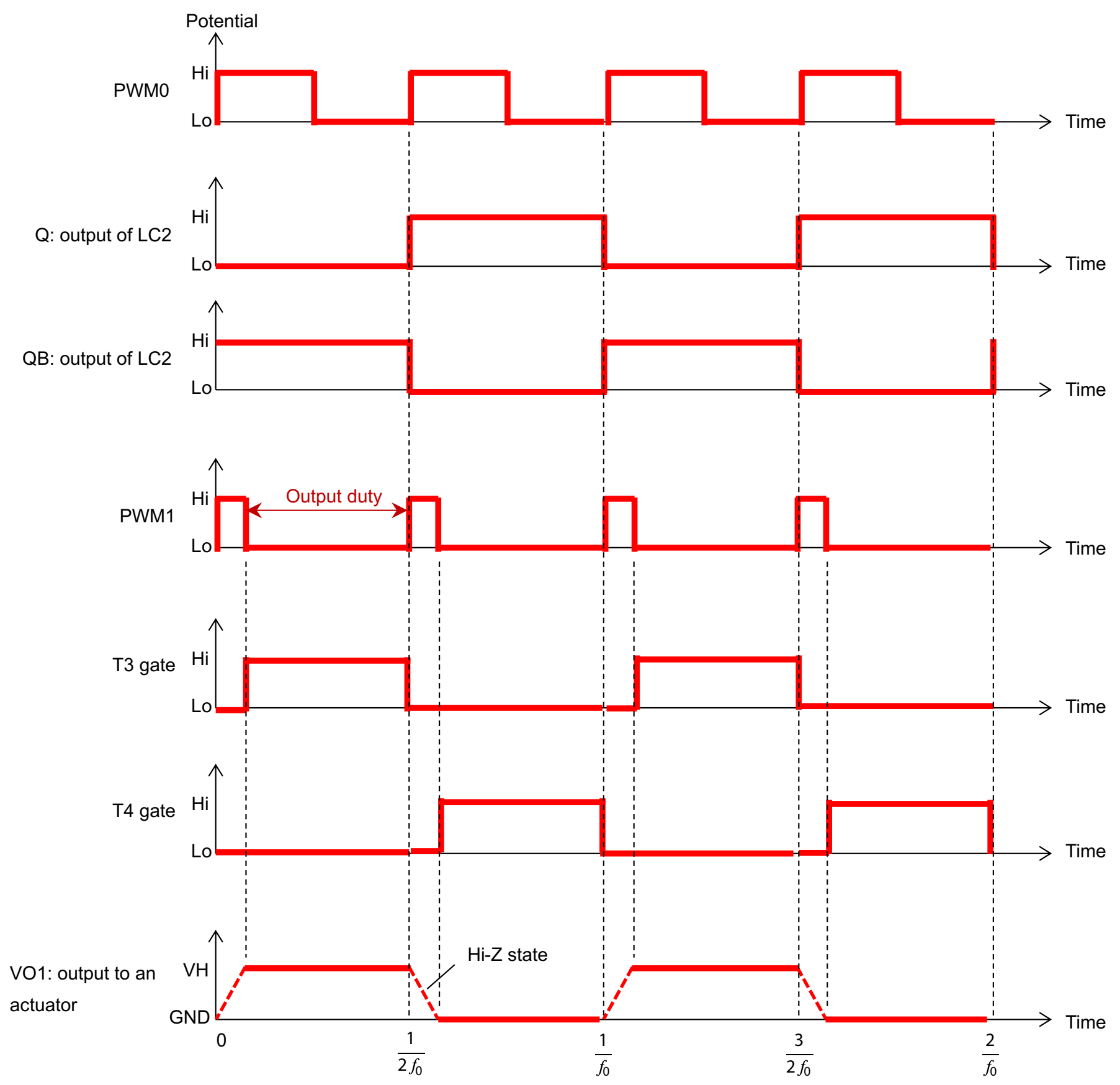

Extended Data Fig. 3 | Driving waveform of high voltage driver. Driving waveform of high voltage driver. Here, as an example, we show the drive waveform for one channel. The square waves $Q$ and QB are generated from PWMO by FF (LC1) with double period. The logical NOR of PWM1 and Q creates the gate voltage of T3 that drives the high-side switch. The gate voltage of T4, which drives the low-side switch, is generated by the logical NOR of PWM1 and QB. When the switch timing of T3 and T4 overlaps, a large loss occurs due to the through current flow from the high voltage side to GND. Therefore, a high impedance period (the period when both T3 and T4 are low) is provided for the transition from the high voltage side to the GND side of VO1. 


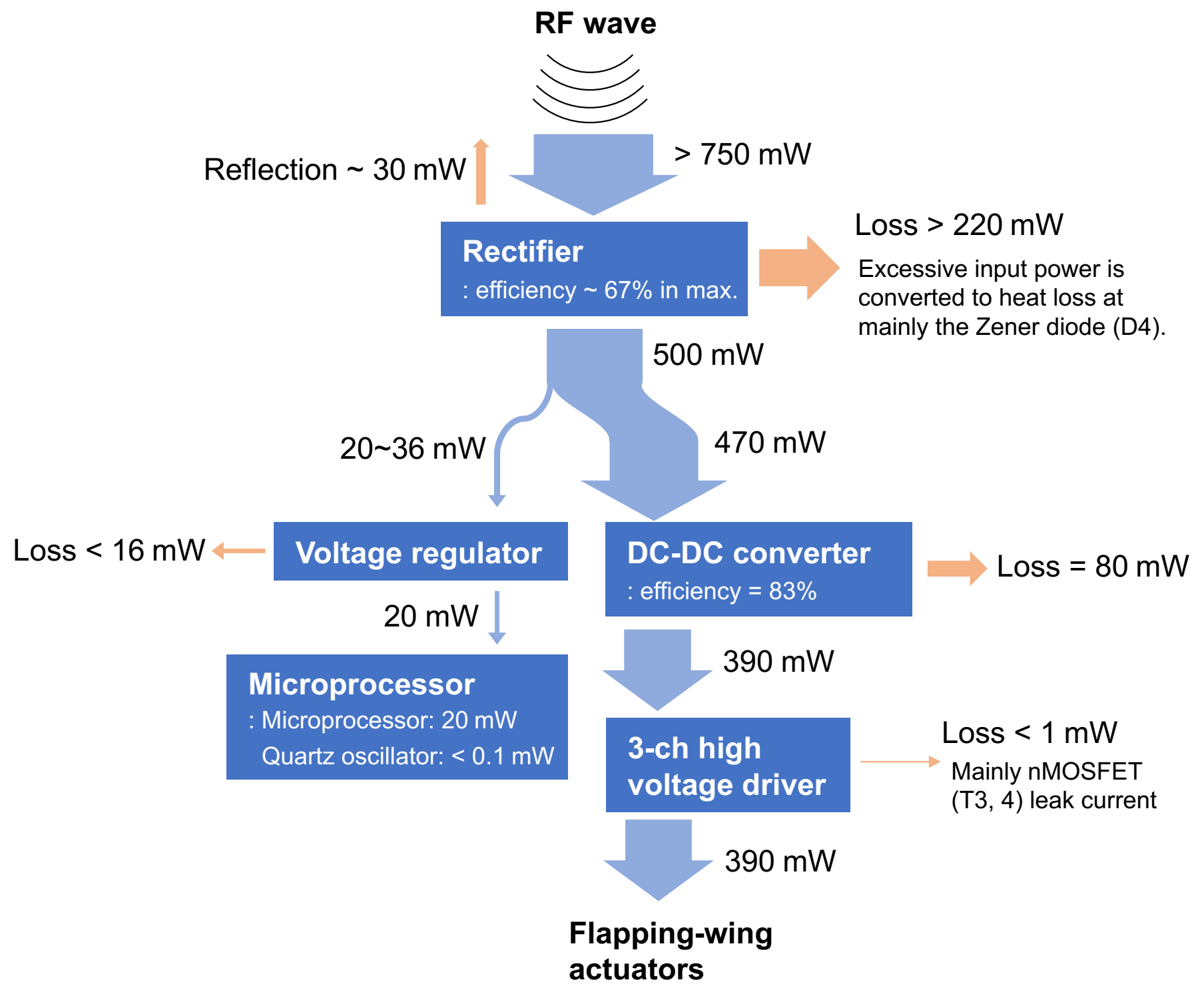

Extended Data Fig. 4 | Power flow diagram in continuous flight. Power flow diagram in continuous flight. 

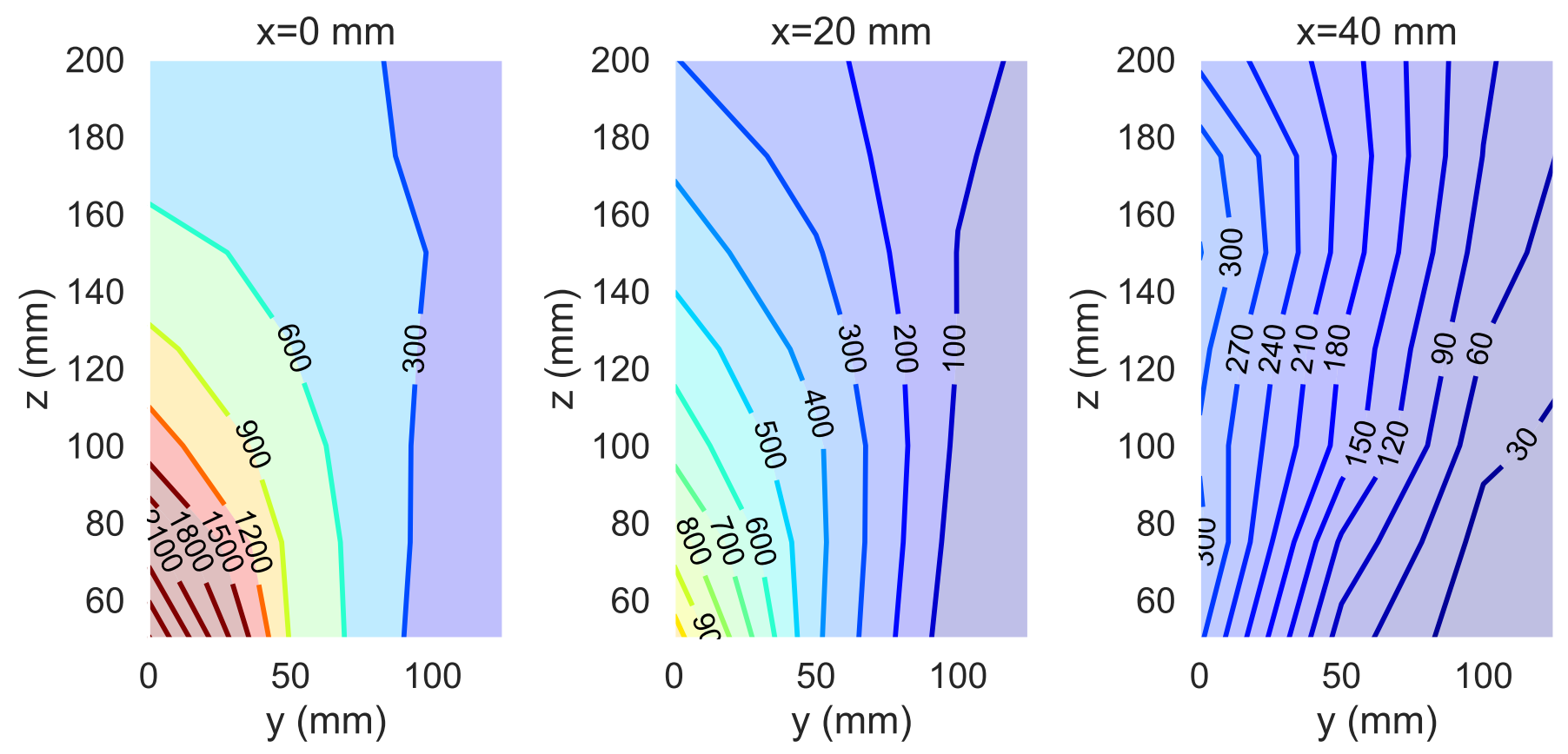

Extended Data Fig. 5 | Three-dimensional distribution of received power. The measurement setup and condition are same as in Fig. 4b. The numbers labelled to the contour map show the received power in $\mathrm{mW}$. 

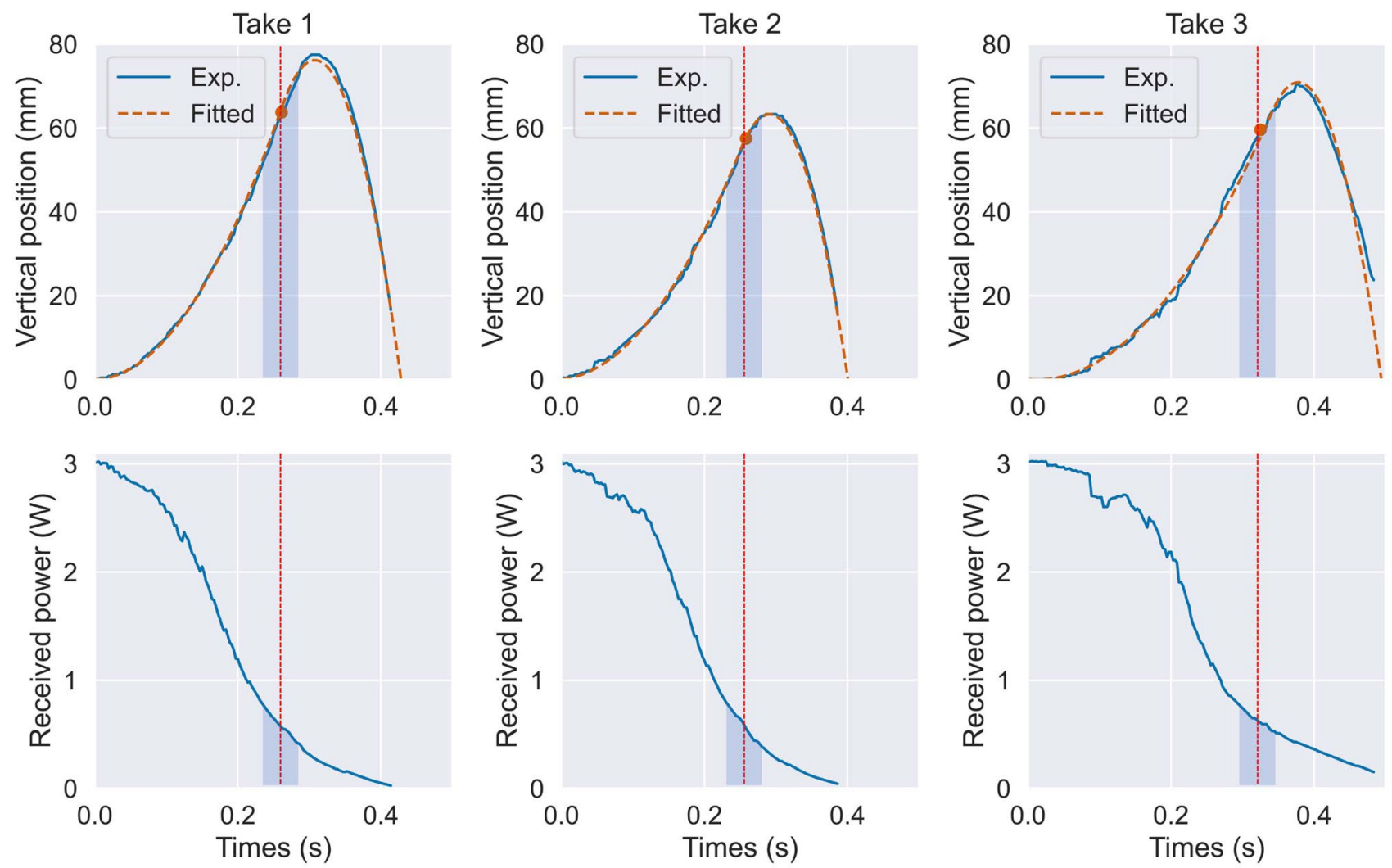

Extended Data Fig. 6 | Fitting result of flight trajectory and estimated received power along the trajectory. Fitting result of flight trajectory and estimated received power along the trajectory. We considered the trajectory to be a quadratic curve both during the ascent and after the actuator stops, and the two quadratic curves that are continuously connected are considered to switch at a certain time $T_{i}$. In other words, the following functions were used to fit the three trajectories using the least-squares method: $z=\left\{\begin{aligned} & \frac{1}{2} a t^{2}: t<T_{i} \\ & \frac{1}{2} g t^{2}+(a-g) T_{i} t-\frac{1}{2}(a-g) T_{i}^{2}: t \geq T_{i}\end{aligned}\right.$ where $a$ and $T$ are the acceleration during ascending and time of the inflection point; they are the fitted parameters. $g$ is the gravitational acceleration: $-9.8 \mathrm{~m} / \mathrm{s}^{2}$. As a result, we obtained the fitting parameters of $\left(a, T_{i}\right)=\left(1.86 \mathrm{~m} / \mathrm{s}^{2}, 0.26 \mathrm{~s}\right),\left(1.62 \mathrm{~m} / \mathrm{s}^{2}, 0.26 \mathrm{~s}\right)$ and $\left(1.18 \mathrm{~m} / \mathrm{s}^{2}, 0.33 \mathrm{~s}\right)$ for the three takes, respectively. The fitting was performed using the 'curve_fit' function in the Python module Scipy. The upper graphs in the figure show the trajectory measurement results superimposed on the fitting results. The red circles are the inflection point: that is, the moment the circuit was estimated to have stopped. Here, we use light blue bands to represent the confidence interval of the shutdown time. In our system, it takes up to 52 ms to shut down due to the energy stored in the capacitor (C2) and the decay time of the actuator when the receiver power becomes insufficient low. The total capacitance is $400 \mu \mathrm{F}$, the maximum receiving voltage is $6 \mathrm{~V}$, the minimum operating voltage of the microprocessor is $1.8 \mathrm{~V}$, and the steady-state consumed power is $500 \mathrm{~mW}$. Therefore, a maximum margin of $400 \mu \mathrm{F} \times\left((6 \mathrm{~V})^{2}-(1.8 \mathrm{~V})^{2}\right) / 500 \mathrm{~mW}=26 \mathrm{~ms}$ can be expected. The Q-factor of the actuator is approximately 3 . Therefore, the amplitude decay time is roughly $(115 \mathrm{~Hz})^{-1} \times 3=26 \mathrm{~ms}$, and intervals of $52 \mathrm{~ms}$ centered on the estimated inflection point are indicated in the graph. From this interval, the confident shutdown altitudes are 53.9-74.5 mm, 48.0-63.2 mm, and 49.5-67.8 mm for takes 1-3, respectively. By linear interpolating the distribution of the received RF power data shown in Extended Data Fig. 5, we estimate the received power along the trajectory. The results were shown in the lower graphs in the figure. The estimated received powers at the inflection points were 549,589, and $652 \mathrm{~mW}$. Similar to the above, considering the confidence interval of $52 \mathrm{~ms}$, the received power at shutdown for takes 1-3 are 390-741 $\mathrm{mW}, 351-743 \mathrm{~mW}$, and 507-724 $\mathrm{mW}$, respectively. 


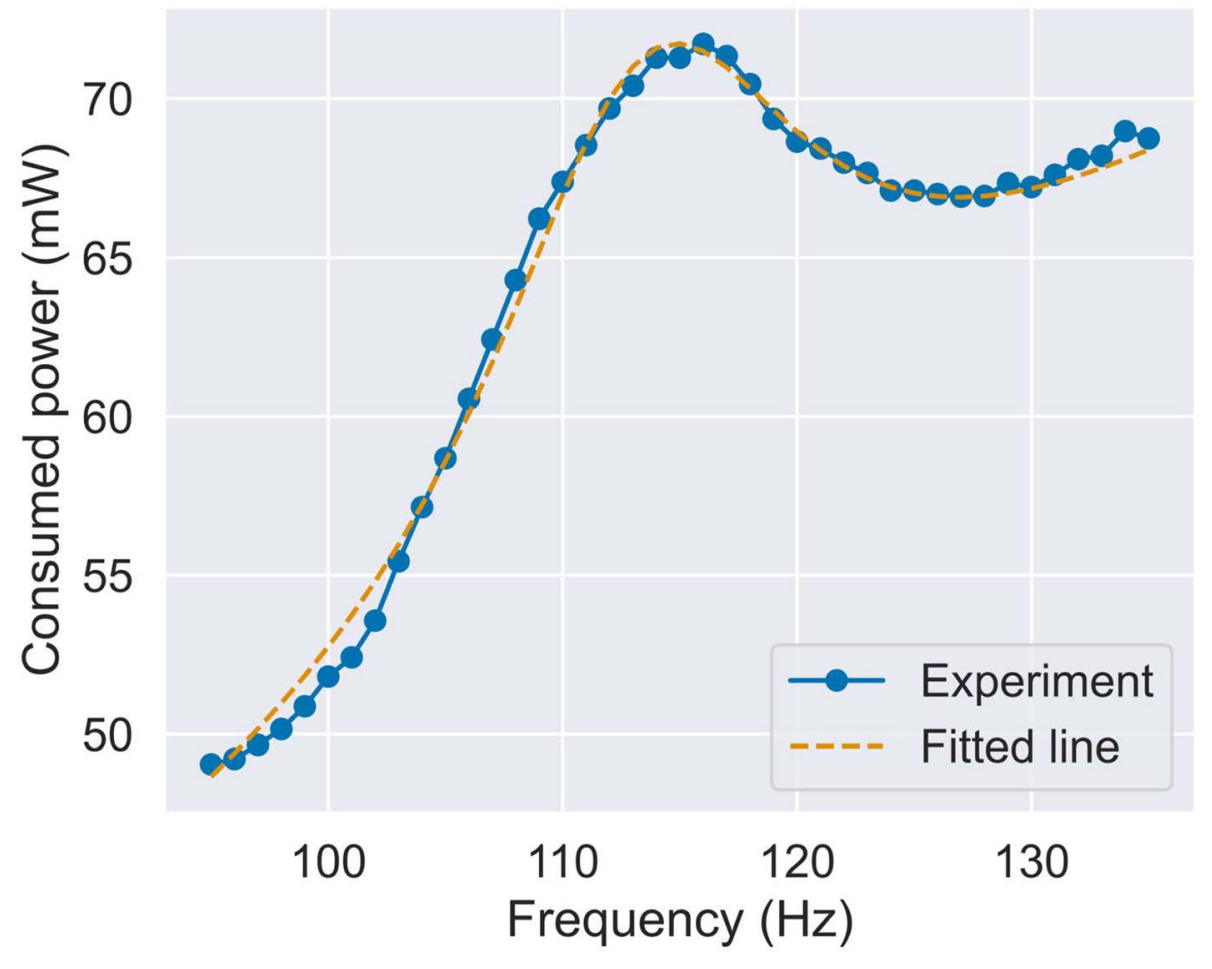

Extended Data Fig. 7 | Frequency-consumed power characteristic of paired-actuator. Frequency-consumed power characteristic of paired-actuator. The parameters of the pseudo-load were estimated from the frequency vs. power consumption characteristics at $50 \%$ duty cycle, $100 \mathrm{~V}$ square wave drive (without charge recovery). The power consumption $P(\omega)$ can be obtained from the Fourier transform of the square wave as follows. $P(\omega)=C_{0} V^{2} \frac{\omega}{2 \pi}+\sum_{k=0} P_{\text {act }}\left((2 k+1) \omega, \frac{\sqrt{2}}{(2 k+1) \pi} V\right), P_{\text {act }}(\omega, V)=\frac{V^{2} R \omega^{2}}{R^{2} \omega^{2}+L^{2}\left(\omega^{2}-(L C)^{-2}\right)^{2}}$, where $R, L$ and $C$ are the RLC circuit parameters for simulating the mechanical characteristics of the actuator. $C_{0}$ is the capacitive component of the actuator. $\omega$ and $V$ are the frequency and voltage amplitude of the drive signal. By fitting this function to the measurement results, the following parameters were obtained: $\left(R, L, C, C_{0}\right)=(127 \mathrm{k} \Omega, 1215 \mathrm{H}, 1.61 \mathrm{nF}, 48.8 \mathrm{nF})$. 


\section{Extended Data Table 1 | Comparison of RF-powered MAVs}

\begin{tabular}{lllll} 
Ref. & Field region & Receiver & Based MAV product & Total mass \\
\hline 30 & Near-field & Inductive coupling coil & H107L X4 (Hubsan) & $50 \mathrm{~g}^{\star}$ \\
31 & Near-field & Inductive coupling coil & JJRC-H98 (Jianjian Technology) & $85 \mathrm{~g}^{\star}$ \\
32 & Near-field & Inductive coupling coil & H107L X4 (Hubsan) & $50 \mathrm{~g}^{\star}$ \\
33 & Near-field & Inductive coupling coil & X525 (hobbypower) & $1.337 \mathrm{~kg}$ \\
34 & Near-field & Inductive coupling coil & Phantom 3 (DJI) & $1.28 \mathrm{~kg}^{\star}$ \\
This work & Far-field & Dipole antenna & - & $1.8 \mathrm{~g}$ \\
\hline
\end{tabular}

* Estimated from the based MAV products 
Extended Data Table 2 | Mass of fabricated circuit

Subcircuit

Power receiver

DC-DC converter

3-ch high voltage driver

Microprocessor
Mass (mg)

Components

60

Substrate and solder ${ }^{\star}$

185

Total

245

Components

51

Substrate and solder*

38

Total

89

Components

70

Substrate and solder ${ }^{\star}$

28

Total

98

Components

28

Substrate and solder*

50

Total

510

* The masses of "substrate and solder" are derived by subtracting the mass of the components from the total mass. 
Extended Data Table 3 | Components of fabricated circuit

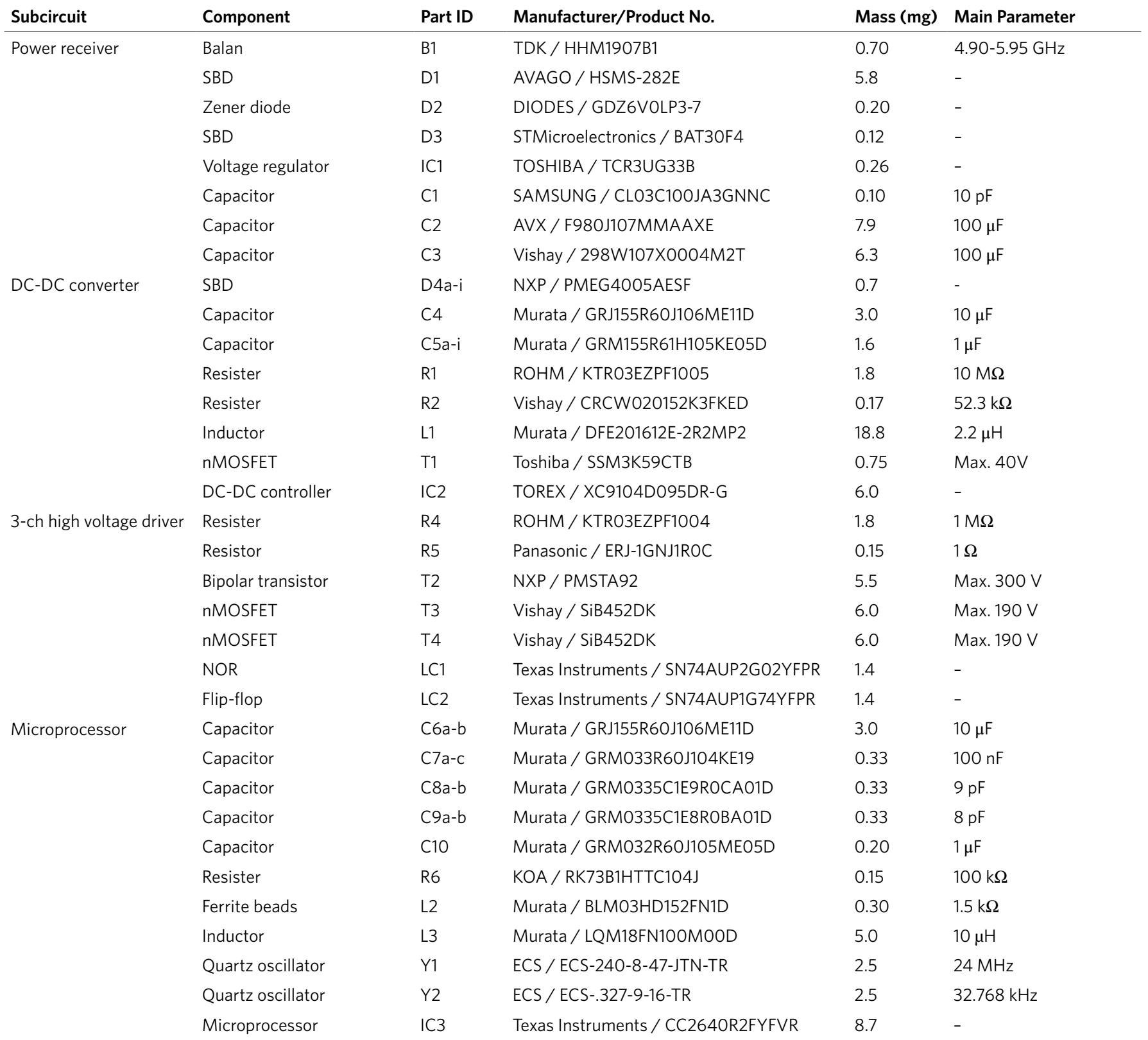

